\title{
Discogenic Axial Back Pain: Is There a Role for Nucleoplasty?
}

\author{
Naresh Satyanarayan $\operatorname{Kumar}^{1}$, Siddharth M Shah ${ }^{2}$, Barry Wei Loong Tan ${ }^{1}$, Salam Juned ${ }^{2}$, Kaihan Yao ${ }^{1}$ \\ ${ }^{1}$ Department of Orthopaedic Surgery, University Spine Centre, National University Hospital, Singapore \\ ${ }^{2}$ Department of Orthopaedic Surgery, National University Hospital, Singapore
}

\begin{abstract}
Study Design: A prospective observational study.
Purpose: To evaluate the role of nucleoplasty in the management of discogenic axial back pain; to determine the influence of concordant pain during provocative discography, annular tear and loss of disc height on the outcome of nucleoplasty.

Overview of Literature: The role of nucleoplasty in the management of radicular leg pain due to disc herniation is known. However, the data regarding its role in the management of discogenic axial back pain is scarce.

Methods: A prospective evaluation of 30 patients with discogenic axial back pain undergoing nucleoplasty was performed. Pain, functional disability and quality of life were assessed using the $100 \mathrm{~mm}$ visual analogue scale (VAS), Oswestry Disability Index (ODI) and Short Form-36 (SF-36), respectively.

Results: The mean reduction in VAS was 31.03 and 29.03; mean reduction in ODI was 24.53 and 20.60; and mean increment in SF-36 was 13.58 and 12.30, at 6 months and at 12 months, respectively. The differences were statistically significant $(p<0.05)$. Concordant pain during provocative discography, annular tear and loss of disc height did not affect a clinically significant improvement in any of the three outcomes $(p=0.882,0.213$, and 0.170 ; respectively).

Conclusions: Nucleoplasty produced statistically significant improvements in pain, functional disability and quality of life in patients with discogenic low back pain at 6 months and at 12 months. Concordant pain during provocative discography, annular tear and loss of disc height did not influence any of the outcomes after nucleoplasty in patients with discogenic axial back pain.
\end{abstract}

Keywords: Nucleoplasty; Radiofrequency treatment; Axial back pain; Degenerate disc disease

\section{Introduction}

Low back pain has a worldwide prevalence of $30 \%$ to $40 \%$ $[1,2]$. The total costs due to low back pain in the United States exceed $\$ 100$ billion per year [3]. Approximately $40 \%$ of all lower back pain has been found to be discogenic in origin [4]. Discogenic low back pain may result from a disc prolapse or degenerative disc disease (DDD). Successful outcomes after discectomy for disc prolapse have been reported [5,6]. However, the patients with small, contained degenerated discs have been found to be poor candidates for discectomy, as their clinical outcomes are less favorable [7].

Discogenic low back pain from DDD has been traditionally managed with non-operative methods or by the major surgical interventions such as spinal fusion. In recent years, several minimally invasive techniques have been developed to treat discogenic low back pain. These

Received Jul 7, 2012; Revised Aug 4, 2012; Accepted Aug 8, 2012

Corresponding author: Naresh Satyanarayan Kumar

University Spine Centre, University Orthopaedics, Hand and Reconstructive Microsurgery Cluster,

1E Kent Ridge Road, NUHS Tower Block, Level 11, Singapore 119228

Tel: +65-6772-5611, Fax: +65-6778-0720, E-mail: dosksn@nus.edu.sg 
techniques aim at reducing the intradiscal pressure and thus decompress the disc, which in turn relieves pressure on the nervous tissue [8]. The treatment approach for the low back pain secondary to DDD is highlighted in Fig. 1.

Nucleoplasty is a minimally invasive technique that was first approved by the US Food and Drug Administration in 2000 [9]. It aims to achieve percutaneous disc decompression through a patented coblation technology, which utilizes the bipolar radiofrequency energy to ablate and remove the disc material, with a coagulation of the adjacent residual disc tissue. There is weak evidence that nucleoplasty is effective in the treatment of radicular leg pain due to contained disc herniation. However, there is no evidence available with regards to its role in managing discogenic axial back pain [10].

Thus, the aim of our study was: 1) to evaluate the role of nucleoplasty in the management of discogenic axial back pain, which failed conservative treatments; 2 ) to determine the influence of concordant pain during provocative discography, presence of annular tear and loss of disc height on the outcome of nucleoplasty.

\section{Materials and Methods}

The Institutional Review Board's approval was obtained prior to the commencement of the study. Patients presenting to the senior author (N.S.K) with discogenic axial back pain were identified. The discogenic nature of the problem was ascertained clinically and radiologically. The patients who satisfied the criteria detailed in Table 1 were offered nucleoplasty for their symptoms. They were also informed that the discography would precede the nucleoplasty procedure. All the patients who accepted nucleoplasty for their symptoms were included in the study. Between October 2008 and December 2009, 30 such patients were recruited and followed up prospectively. None of the patients were lost to the follow-up at 12 months. Magnetic resonance imaging (MRI) of the recruited patients were analyzed for the following: the level at which the degenerate discs were present; severity of the disc degeneration according to the Pfirman's grading [11]; and the presence of annular tear and loss of disc height. All patients were assessed for pain, functional disability and quality of life using $100 \mathrm{~mm}$ visual analogue scale (VAS),

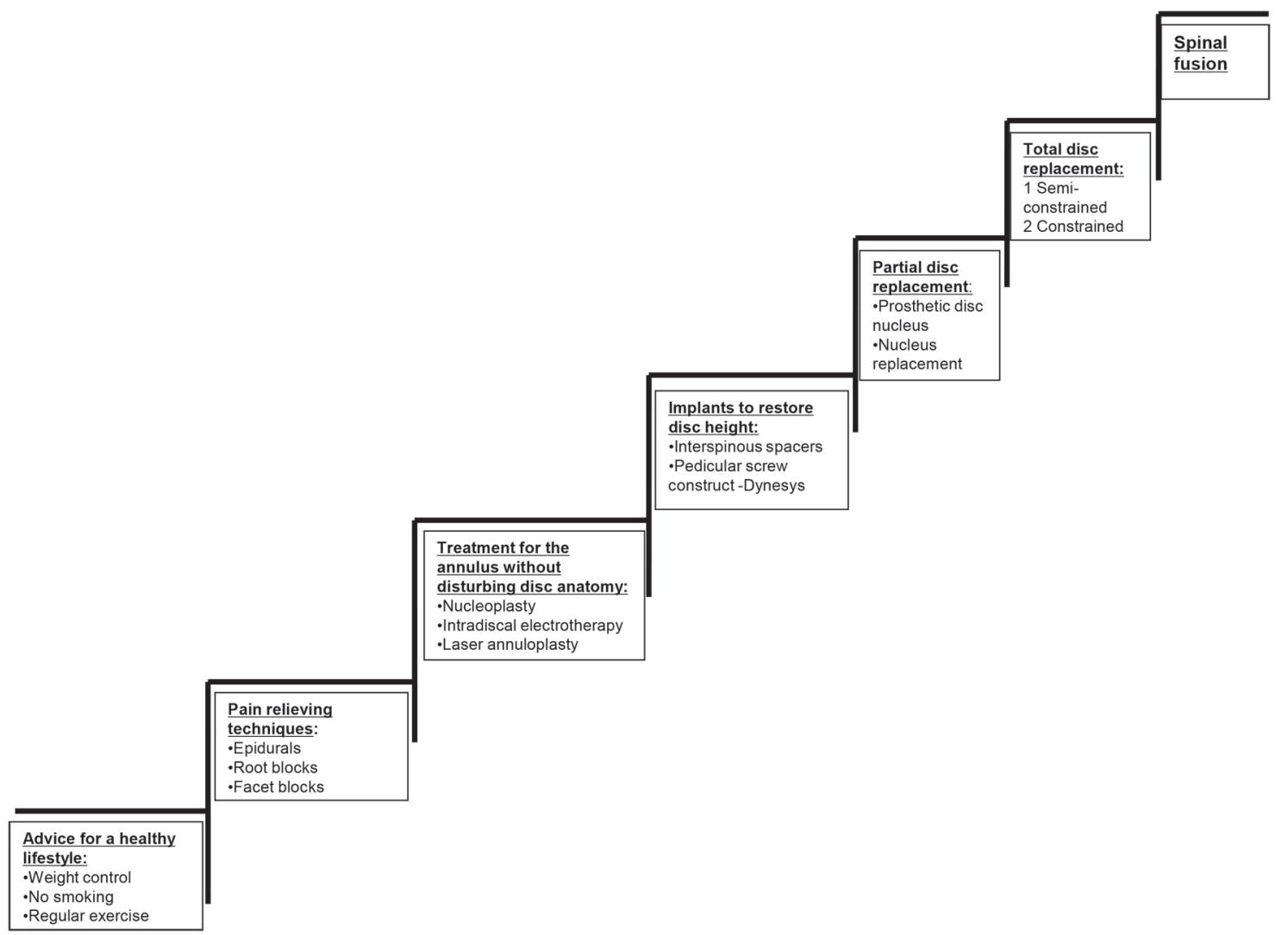

Fig. 1. Stepladder treatment approach for management of pain from degenerative disc disease. 
Table 1. Inclusion and exclusion criteria

\section{Inclusion criteria}

Age between 18 and 60 years

Axial back pain, with or without non- dermatomal lower limb pain

Pain must be significant enough to interfere with daily activities

Patient must have failed at least 6 months of conservative treatment including two or more of the following-analgesics, muscle relaxants, core strengthening exercises, heat therapy and acupuncture

Accurate identification of the symptomatic disc level prior to the procedure

The disc in question falls within Pfirrmann grade 2 to 4 on preprocedure magnetic resonance imaging
Exclusion criteria

Radicular leg pain

Spinal instability

Sequestrated disc

Previous lumbar spine surgery

Discitis

Litigation

Psychiatric illness

Facet arthritis

Moderate to severe canal stenosis

Degenerated discs at more than 2 contiguous levels
Oswestry Disability Index (ODI) and Short Form-36 (SF36), respectively, prior to the procedure.

\section{Procedure}

All patients were managed in the short-stay ward. A prophylactic dose of $1 \mathrm{~g}$ cefazolin was administered intravenously 1 hour prior to the procedure. Discography preceded nucleoplasty in every case. With the patient in a prone position, the procedure was carried out under local anesthesia with sedation and under the image intensifier guidance. A 17-gauge needle was introduced via a posterolateral approach towards the identified disc level and advanced into the central third of the disc. After confirming the position of the needle tip, a nonionic contrast dye (Omnipaque 350, GE Healthcare, Cork, Ireland) was injected into the disc. Concordance of pain was noted at the level in question and was validated with a control disc. After the discography, a coblation bipolar device (Perc DLG SpineWand connected to ArthroCare System 2000, Arthrocare, Sunnyvale, CA, USA) was inserted into the disc through the same needle. Disc decompression was achieved through 6 standard coblation channels, which were created by advancing the coblation bipolar device in the ablation mode and retracting it in the coagulation mode. After the withdrawal of the coblation bipolar device, $2 \mathrm{~mL}$ of $0.25 \%$ bupivacaine was injected into the nucleoplasty tract, but not intradiscally. All patients were observed for 24 hours postoperatively for any development of neurological deficit or other procedure-related problems.

Periodic reviews were performed at 6 weeks, 3 months, 6 months, and 12 months. At the 6 months and 12 months follow-up, patients were assessed for the residual and/or new symptoms, and their outcome scores were evaluated.

The minimum clinically important difference (MCID) for each of the three clinical outcome indicators was defined as follows: 1) A decrease in VAS score by 30 points or more [12]. 2) A decrease in ODI score by 12.8 points or more [13]. 3) An increase in SF-36 score by 5 points or more [13].

The overall clinical outcome was defined as: Excellent, clinically significant improvement in all three clinical outcome indicators (VAS, ODI, SF-36); Good, clinically significant improvement in any two outcome indicators; Fair, clinically significant improvement in any one outcome indicator; and Poor, no clinically significant improvement in any of the outcome indicators.

\section{Statistical analysis}

Patient's demographic and baseline clinical data were analyzed descriptively. The VAS, ODI, and SF-36 scores at 6 months and 12 months after the nucleoplasty procedure were compared with the respective pre-procedure scores using paired $t$-test. The mean reduction in VAS and ODI scores and mean improvement in SF-36 scores were estimated, and the point estimates with $95 \%$ confidence interval (CI) were reported. The influence of concordant pain during provocative discography, presence of annular tear and loss of disc height on the clinically significant improvement in any of the three outcomes (VAS, ODI, and SF-36) were evaluated, using the generalizing estimating equations (GEE) model. All statistical analyses were performed using SPSS ver. 18.0 (SPSS Inc., Chicago, 
IL, USA).

\section{Results}

\section{Patient demographics}

A total of 30 patients were recruited for the study, which included $25(83.30 \%)$ males and $5(16.70 \%)$ females. The mean age of the study population was 35 years (range, $20-57$ years). Nineteen $(19 / 30,63.30 \%)$ patients had nondermatomal lower limb pain along with axial back pain. Majority of the patients $(23 / 30,76.70 \%)$ were symptomatic for more than 12 months. Fifteen (50\%) patients had a loss of disc height, and 11 patients (36.70\%) had annular tears at the disc level in question on the preoperative MRI. Nucleoplasty was performed at single level in 24 patients and at two levels in six patients. Thus a total of 36 discs were treated as follows: L2-3 disc, one; L3-4 disc, one; L4-5 disc, 13; and L5-S1 disc, 21. Concordant pain during discography was seen in $19(19 / 30,63.33 \%)$ patients.

\section{Pain reduction (VAS)}

Clinically significant pain reduction was seen in 53.30\% and $60 \%$ of the patients at 6 months and 12 months after the procedure, respectively (Fig. 2). The mean reduction in VAS score was 31.03 (95\% CI, 26.73-35.34, $p<0.05$ )

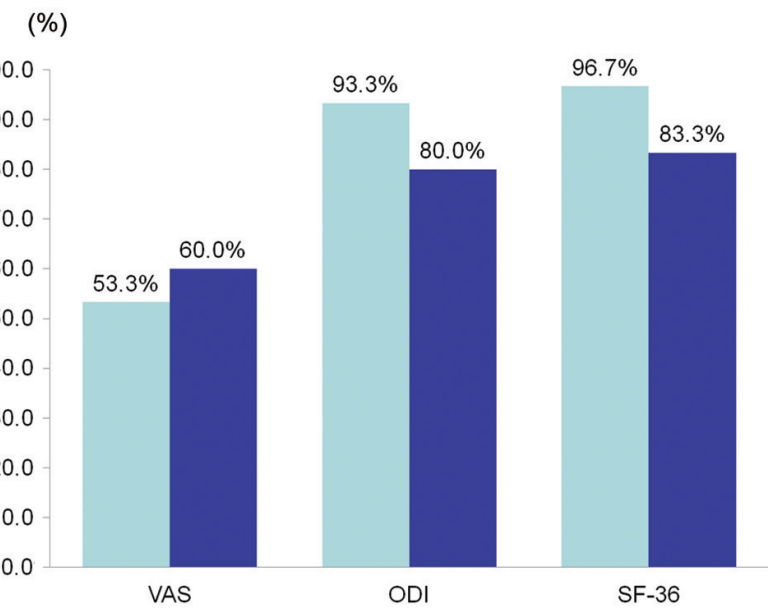

Fig. 2. Graph showing the percentage of patients who achieved a clinically significant improvement in the three clinical outcome indicators (VAS, ODI, and SF-36) at 6 months and 12 months after nucleoplasty. VAS, visual analogue scale; ODI, Oswestry Disability Index; SF-36, Short Form-36. and 29.03 ( $95 \%$ CI, $23.66-34.40, p<0.05$ ) at 6 months and 12 months, respectively (Fig. 3). The scores indicated a statistically, but not clinically, significant mean improvement. The baseline VAS score significantly influenced the post procedure improvement in the VAS score $(p=0.010)$.

\section{Functional disability reduction (ODI)}

Clinically significant reduction in the functional disability at 6 months and 12 months was seen in $93.30 \%$ and $80 \%$ of patients, respectively (Fig. 2). The mean reduction in ODI score was 24.53 (95\% CI, 21.15-27.92; $p<0.05)$ and 20.600 (95\% CI, $16.90-24.30 ; p<0.05$ ) at 6 months and 12 months, respectively (Fig. 3). The mean reduction in ODI score was both statistically and clinically significant. Baseline ODI score did not influence the post procedure improvement in the ODI score significantly $(p=0.109)$.

\section{Improvement in quality of life (SF-36)}

Clinically significant improvement in quality of life at 6 months and 12 months was seen in $96.70 \%$ and $83.30 \%$ of patients, respectively (Fig. 2). The mean increment in the SF-36 score was 13.58 (95\% CI, $11.41-15.75 ; p<0.05)$ and $12.30(95 \% \mathrm{CI}, 9.50-15.11 ; p<0.05)$ at 6 months and 12 months, respectively (Fig. 3). The scores indicated both statistically and clinically significant mean improvements. The baseline SF-36 score did not influence the post procedure SF-36 score significantly $(p=0.590)$.

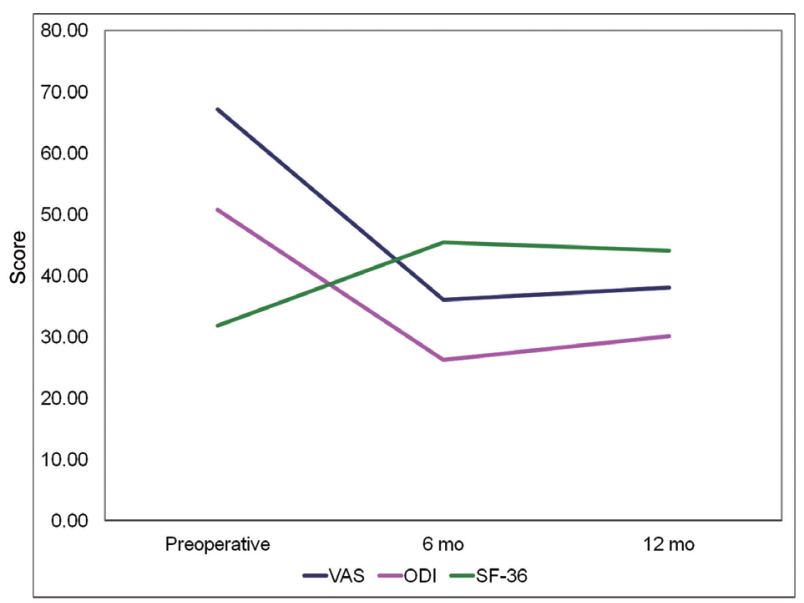

Fig. 3. Graph showing mean scores of the three clinical outcome indicators (VAS, ODI, and SF-36) at preoperative, 6 months, and 12 months follow-up period. VAS, visual analogue scale; ODI, Oswestry Disability Index; SF-36, Short Form-36. 


\section{Correlation between other variables and clinical out- come after nucleoplasty}

The GEE model demonstrated that none of the following variables-concordant pain during discography, presence of annular tear or presence of loss of disc height-influenced clinically a significant improvement in any of the 3 outcomes ( $p=0.882,0.213$, and 0.170 ; respectively). However, the patients with a loss of disc height improved 7.5 points more in ODI ( $95 \%$ CI, $0.50-10.38, p<0.05)$, compared to the patients with preserved disc heights, across both time points.

There was no significant change in the clinically significant improvements achieved in all 3 outcomes at the end of 6 months and 12 months ( $p=0.478$ ).

While assessing the overall clinical outcomes, Good to Excellent results were seen in 28 patients (28/30, 93.33\%) at the end of 6 months and were sustained in 22 patients $(22 / 30,73.33 \%)$ at the end of 12 months.

\section{Discussion}

The biomechanical and biochemical effects of nucleoplasty on the intervertebral discs have been well established. Chen et al. [14] showed in a human cadaveric study that nucleoplasty reduced the intradiscal pressures significantly, especially in the younger, healthy discs. In 2003, Chen et al. [15] demonstrated the safety of nucleoplasty in a porcine model. He showed that the procedure achieved a volumetric reduction of the target disc tissues without overt thermal or structural damages to the adjacent tissues. Lee et al. [16] reconfirmed the safety of nucleoplasty in the sheep intervertebral discs. In 2004, O'Neill et al. [17] demonstrated the significant biochemical changes in the degenerate porcine discs after nucleoplasty in the form of a decrease in Interleukin-1 and increase in interleukin-8. These changes were consistent with the mechanism of pain relief.

Several studies have found favorable results with nucleoplasty in the treatment of leg pain without axial symptoms. Bokov et al. [18] found that nucleoplasty provided a significant relief of leg pain and faster recovery compared to microdiscectomy in patients with contained disc herniations. Mirzai et al. [19] prospectively evaluated the results of nucleoplasty in 52 patients with disc herniations causing leg pain, with respect to pain reduction, patient satisfaction, functional disability and anal- gesia requirement. They reported a mean reduction in pain by $>50 \%$, patient satisfaction $>80 \%$ and good results in the functional disability improvement and analgesia requirement. Gerszten et al. [20], in their study of 67 patients with primary radicular leg pain due to disc herniations, concluded that nucleoplasty is a safe and viable minimally-invasive technique that improved leg pain and quality of life in patients with contained disc herniations. In contrast, a smaller study on 16 patients with leg pain by Cohen et al. [21] concluded that nucleoplasty was not an effective treatment of lumbar radiculopathy, as the mean pain scores did not improve significantly and only one patient achieved $>50 \%$ reduction in the VAS score. Despite these encouraging studies, it is imperative to note that nucleoplasty is still not considered a substitute for microdiscectomy and is suitable for patients with the relatively small and contained disc herniations.

In addition to the use of nucleoplasty in the pure radicular leg pain, several studies have included axial back pain along with leg pain in the inclusion criteria. In 2002, Singh et al. [22] studied 67 patients with contained disc herniations and concluded that nucleoplasty is effective in treating discogenic back and/or leg pain in patients who failed conservative management and are not candidates for open surgery. Their conclusion was based on significant improvements at 12 months in pain and function. Al-Zain et al. [23] conducted a prospective study on 96 similar patients treated with nucleoplasty and found that $73 \%$ of the patients achieved a $>50 \%$ improvement in the VAS score at the early follow-up, with a decrease in the analgesia requirement, disability and work incapacitation. However, the improvements in the outcomes decreased over time.

There is a paucity of literature focusing on the success of nucleoplasty in the treatment of the isolated discogenic axial back pain. In 2004, Singh et al. [24] published a prospective study involving 47 patients with predominantly axial back pain secondary to disc herniation, treated with a nucleoplasty and followed up for 12 months. They found significant improvements in pain and function at all follow-ups, although the ratio of patients showing significant improvements decreased over time. Our study endeavors to provide further evidence substantiating the role of nucleoplasty in the treatment of isolated discogenic axial back pain.

The effectiveness of nucleoplasty in the treatment of discogenic axial back pain is demonstrated in our results. 
Statistically significant improvements in VAS, ODI and SF-36 scores were seen at 6 months and 12 months. In addition, a majority of our patients achieved MCID in each of the three clinical outcome indicators as defined by us. Good to excellent results were seen in $93.33 \%$ and $73.33 \%$ patients at the end of 6 months and 12 months, respectively. Our findings contrast with those of Gerges et al. [9], who have reported that nucleoplasty may be less effective for patients with discogenic back pain without radicular symptoms.

Furthermore, a clinically significant improvement in ODI was seen in $93.30 \%$ patients at 6 months and $80 \%$ patients at 12 months. Similarly, a clinically significant improvement in SF-36 was demonstrated in $96.70 \%$ patients at 6 months and $83.30 \%$ patients at 12 months. Although all patients demonstrated some degree of improvement in the VAS score, the ratio of patients achieving a clinically significant improvement was lower for VAS score $(53.30 \%$ at 6 months and $60 \%$ at 12 months) as compared to the ODI and SF-36 scores. In addition, a clinically significant mean improvement was not sufficiently demonstrated for the VAS scores in our study as per the MCID defined by us. A larger sample size may provide a more conclusive evidence of a clinically significant improvement in the VAS scores after nucleoplasty, based on the values adopted by us. Sharps and Isaac [25] defined a clinically significant improvement in the VAS scores as a reduction in the score by two points. If our study defined the MCID of VAS as 20 points instead of $30,86.70 \%$ of patients would have achieved a clinically significant improvement in the VAS at 6 months, and $76.70 \%$ at 12 months. The mean improvement in the VAS scores would also be clinically significant at 6 months and 12 months.

We noted a slight worsening in all three clinical outcome indicators at 12 months compared to 6 months. This finding is comparable to that of the aforementioned studies [23,24]. Moreover, this slight worsening in scores was not statistically significant.

\section{Role of discography, annular tear and disc height in predicting outcome after nucleoplasty}

Concordant pain during provocative discography has been described by several authors as a predictor of discogenic low back pain and has been used as an inclusion criterion for nucleoplasty [24,25]. Lim et al. [26] conclud- ed that fissured or ruptured discs and the extension of contrast into or beyond the outer annulus on the computed tomography (CT) discography were associated with concordant low back pain. Sharps and Isaac [25] recommended that concordant pain during provocative discography should be an inclusion criterion for nucleoplasty, as these patients had a significant reduction in the VAS scores after nucleoplasty. However our results showed no significant correlation between the concordant pain during discography and a clinically significant improvement in any of the three outcome indicators.

According to Bokov et al. [18], a total annulus disruption significantly influences the results of nucleoplasty. Patients with a total annulus disruption compared to those with contained disc herniation showed a significant decrease in the success rate and total pain relief. Our results, however, demonstrate that the annular integrity may not be a critical factor in determining the success of nucleoplasty, as there was no significant correlation between the annular tear and clinically significant improvement in any of the three clinical outcome indicators.

Chen et al. [14] found that the change in the intradiscal pressure after nucleoplasty was negligible in the older and degenerated discs with loss of disc height, whereas intradiscal pressure was markedly reduced in the younger and healthy discs. Based on this study and the postulated mechanism of action of nucleoplasty in the treatment of axial back pain, Mirzai et al. [19] utilized the disc height of $\geq 50 \%$ as an inclusion criterion for nucleoplasty. In our study, patients with a loss of disc height showed a statistically greater improvement in the ODI scores across both time points compared to patients with preserved disc heights.

A small sample size and short follow-up are the drawbacks of our study. Nevertheless, our study does offer some significant findings. By not limiting our inclusion criteria to only those discs that show pain concordance, relatively preserved disc heights and an intact annulus, we have been able to show that these factors do not influence the outcome of nucleoplasty. In other words, the benefits of nucleoplasty may be extended to a greater proportion of patients with discogenic axial back pain that fail conservative treatments.

\section{Conclusions}

Nucleoplasty produced statistically significant improve- 
ments in pain, functional disability and quality of life in patients with discogenic axial back pain after failed conservative treatments at 6 months and 12 months. Clinically significant improvements were seen in the functional disability and quality of life at both 6 months and 12 months after nucleoplasty. Good to excellent results were seen in the majority of the cases at 12 months. Concordant pain during provocative discography, presence of annular tear and loss of disc height did not influence any of the outcomes after nucleoplasty in patients with discogenic axial back pain. Nucleoplasty may benefit a larger proportion of patients with discogenic axial back pain, if the inclusion criteria are appropriately modified.

\section{Conflict of Interest}

No potential conflict of interest relevant to this article was reported.

\section{References}

1. Papageorgiou AC, Croft PR, Ferry S, Jayson MI, Silman AJ. Estimating the prevalence of low back pain in the general population: evidence from the south manchester back pain survey. Spine (Phila Pa 1976) 1995;20:1889-94.

2. Skovron ML, Szpalski M, Nordin M, Melot C, Cukier D. Sociocultural factors and back pain. A populationbased study in Belgian adults. Spine (Phila Pa 1976) 1994;19:129-37.

3. Katz JN. Lumbar disc disorders and low-back pain: socioeconomic factors and consequences. J Bone Joint Surg Am 2006;88 Suppl 2:21-4.

4. Kallewaard JW, Terheggen MA, Groen GJ, et al. 15. Discogenic low back pain. Pain Pract 2010;10:560-79.

5. Mirza SK, Deyo RA. Systematic review of randomized trials comparing lumbar fusion surgery to nonoperative care for treatment of chronic back pain. Spine (Phila Pa 1976) 2007;32:816-23.

6. Weinstein JN, Lurie JD, Tosteson TD, et al. Surgical versus nonoperative treatment for lumbar disc herniation: four-year results for the Spine Patient Outcomes Research Trial (SPORT). Spine (Phila Pa 1976) 2008;33:2789-800.

7. Dora C, Schmid MR, Elfering A, Zanetti M, Hodler J, Boos N. Lumbar disk herniation: do MR imaging findings predict recurrence after surgical diskectomy?
Radiology 2005;235:562-7.

8. Singh V, Derby R. Percutaneous lumbar disc decompression. Pain Physician 2006;9:139-46.

9. Gerges FJ, Lipsitz SR, Nedeljkovic SS. A systematic review on the effectiveness of the Nucleoplasty procedure for discogenic pain. Pain Physician 2010;13:11732.

10. Manchikanti L, Derby R, Benyamin RM, Helm S, Hirsch JA. A systematic review of mechanical lumbar disc decompression with nucleoplasty. Pain Physician 2009;12:561-72.

11. Pfirrmann CW, Metzdorf A, Zanetti M, Hodler J, Boos N. Magnetic resonance classification of lumbar intervertebral disc degeneration. Spine (Phila $\mathrm{Pa}$ 1976) 2001;26:1873-8.

12. Lee JS, Hobden E, Stiell IG, Wells GA. Clinically important change in the visual analog scale after adequate pain control. Acad Emerg Med 2003;10:112830.

13. Copay AG, Glassman SD, Subach BR, Berven S, Schuler TC, Carreon LY. Minimum clinically important difference in lumbar spine surgery patients: a choice of methods using the Oswestry Disability Index, Medical Outcomes Study questionnaire Short Form 36, and pain scales. Spine J 2008;8:968-74.

14. Chen YC, Lee SH, Chen D. Intradiscal pressure study of percutaneous disc decompression with nucleoplasty in human cadavers. Spine (Phila Pa 1976) 2003;28:661-5.

15. Chen YC, Lee SH, Saenz Y, Lehman NL. Histologic findings of disc, end plate and neural elements after coblation of nucleus pulposus: an experimental nucleoplasty study. Spine J 2003;3:466-70.

16. Lee MS, Cooper G, Lutz GE, Doty SB. Histologic characterization of coblation nucleoplasty performed on sheep intervertebral discs. Pain Physician 2003;6:439-42.

17. O’Neill CW, Liu JJ, Leibenberg E, et al. Percutaneous plasma decompression alters cytokine expression in injured porcine intervertebral discs. Spine J 2004;4:88-98.

18. Bokov A, Skorodumov A, Isrelov A, Stupak Y, Kukarin A. Differential treatment of nerve root compression pain caused by lumbar disc herniation applying nucleoplasty. Pain Physician 2010;13:469-80.

19. Mirzai H, Tekin I, Yaman O, Bursali A. The results of nucleoplasty in patients with lumbar herniated disc: a 
prospective clinical study of 52 consecutive patients. Spine J 2007;7:88-92.

20. Gerszten PC, Welch WC, King JT Jr. Quality of life assessment in patients undergoing nucleoplastybased percutaneous discectomy. J Neurosurg Spine 2006;4:36-42.

21. Cohen SP, Williams S, Kurihara C, Griffith S, Larkin TM. Nucleoplasty with or without intradiscal electrothermal therapy (IDET) as a treatment for lumbar herniated disc. J Spinal Disord Tech 2005;18 Suppl:S119-24.

22. Singh V, Piryani C, Liao K, Nieschulz S. Percutaneous disc decompression using coblation (nucleoplasty) in the treatment of chronic discogenic pain. Pain Physi- cian 2002;5:250-9.

23. Al-Zain F, Lemcke J, Killeen T, Meier U, Eisenschenk A. Minimally invasive spinal surgery using nucleoplasty: a 1-year follow-up study. Acta Neurochir (Wien) 2008;150:1257-62.

24. Singh V, Piryani C, Liao K. Role of percutaneous disc decompression using coblation in managing chronic discogenic low back pain: a prospective, observational study. Pain Physician 2004;7:419-25.

25. Sharps LS, Isaac Z. Percutaneous disc decompression using nucleoplasty. Pain Physician 2002;5:121-6.

26. Lim CH, Jee WH, Son BC, Kim DH, Ha KY, Park CK. Discogenic lumbar pain: association with MR imaging and CT discography. Eur J Radiol 2005;54:431-7. 